Résumés des conférences et travaux

\title{
Histoire de l'architecture et des jardins du Japon
}

\author{
Nicolas Fiévé
}

\section{OpenEdition \\ Journals}

Édition électronique

URL : https://journals.openedition.org/ashp/4903

DOI : $10.4000 /$ ashp.4903

ISSN : 1969-6310

Éditeur

Publications de l'École Pratique des Hautes Études

\section{Édition imprimée}

Date de publication : 1 septembre 2021

Pagination : 482-500

ISSN : 0766-0677

\section{Référence électronique}

Nicolas Fiévé, « Histoire de l'architecture et des jardins du Japon », Annuaire de l'École pratique des hautes études (EPHE), Section des sciences historiques et philologiques [En ligne], 152 | 2021, mis en ligne le 14 juin 2021, consulté le 17 juin 2022. URL : http://journals.openedition.org/ashp/4903 ; DOI : https://doi.org/10.4000/ashp.4903 


\title{
HISTOIRE DE L'ARCHITECTURE ET DES JARDINS DU JAPON
}

\author{
Directeur d'études : M. Nicolas FIÉvé
}

Programme de l'année 2019-2020 : Le château des seigneurs Ii du fief de Hikone, ses palais et ses jardins à l'époque d'Edo. Troisième partie : le palais de Zelkova.

À la fin $\mathrm{du} \mathrm{XVII}^{\mathrm{e}}$ siècle, les seigneurs Ii entretenaient deux résidences dans leur fief de Hikone : le palais officiel, Omote goten 表御殿, bâti à l'intérieur de la première enceinte du château, et le palais de Zelkova, Keyaki goten 槻御殿, une villa de plaisance aménagée le long d'un parc paysager, à l'extérieur de la première enceinte, entre la douve intérieure (uchi-bori 内堀) et le lac Matsubara (Matsubarako 松原湖). La villa de plaisance s'étendait ainsi au pied du château-fort, sur la face nord du Mont Hikone 彦根山 et sur un terre-plein ceint des douves et du lac. Les travaux de construction du palais de Zelkova ont débuté en 1677 (Enpō 5), au temps du 4e seigneur Ii Naooki 井伊直興 (1656-1717) ${ }^{1}$, pour être achevés deux ans plus tard, en 1679 (Enpō 7).

Le plan du palais de Zelkova est connu grâce à plusieurs documents réalisés par les charpentiers qui travaillaient au palais, des documents aujourd'hui conservés aux archives du musée du château de Hikone, Hikone-jo hakubutsukanzō 彦根城博物 館蔵: plans du palais, plans relatifs à la restauration du palais, plans de projets de construction, documents techniques relatifs aux circuits d'alimentation en eau et à l'aménagement des jardins, ainsi que des plans et des coupes des quelques pavillons. L'examen de ces documents montre différentes étapes de l'évolution du palais, jusqu'au remaniement de grande ampleur réalisé en 1812 (Bunka 9) par le $11^{\mathrm{e}}$ seigneur Naonaka 井伊直中 (1766-1831), alors qu'il prenait sa retraite et que Naoaki 直 亮 (1794-1850) devenait le $12^{\mathrm{e}}$ seigneur de Hikone. Quittant sa résidence principale d'Edo, Naonaka est retourné habiter à Hikone en 1813 (Bunka 10), où il lui fallait une demeure indépendante du palais officiel qu'allait désormais occuper son successeur, d'où le réaménagement du palais de Zelkova.

$\mathrm{Du} \mathrm{XVII}^{\mathrm{e}}$ siècle au $\mathrm{XIX}^{\mathrm{e}}$ siècle, la résidence secondaire apparaît dans les sources sous les divers noms de Keyaki goten 梘御殿, “palais de Zelkova », Keyaki gomon goten 梘御門御殿, “palais de la Porte de Zelkova », Kuro gomon soto yashiki 黒 御門外屋敷, 《résidence [bâtie] à l'extérieur de la Porte Noire», ou Kuro gomon mae yashiki 黒御門前屋敷, 《résidence [bâtie] devant la Porte Noire », du nom de la porte de la première enceinte qui, au nord du château, permettait d'accéder directement à la villa.

1. Naooki était alors membre du Conseil des anciens, rôjū charge de Grand ancien, dairô 大老, à deux reprises : de 1697 à 1700, et de 1711 à 1714, mais cette fois-ci sous le nom Naomori 直該. 


\section{Le palais de Zelkova du temps de Naohide, d'après le Keyaki gomon goten oezu de 1756}

Le plan original du palais de 1679 n'est pas connu. Le plus vieux document conservé aux archives du château, le "Plan du Palais de la Porte de Zelkova », Keyaki gomon goten oezu 槻御門御殿御絵図 $(201 \mathrm{~cm} \times 134 \mathrm{~cm})$, est daté de 1756 (Hōreki 6) $)^{2}$ et fut réalisé un an après la nomination de Naohide 直幸 (1729-1789) au titre de $10^{\mathrm{e}}$ seigneur de Hikone. Le document permet ainsi de connaître l'état du palais soixante-dix-sept ans après sa construction et présente le projet de plusieurs bâtiments nouveaux. Croisés à d'autres sources, ces éléments laissent à penser que le plan d'origine ne devait être guère différent de celui de 1756, bien que l'on sache que les dimensions du palais avait été quelque peu réduites du temps du $7^{\mathrm{e}}$ seigneur Naonobu 直惟 (1700-1736).

Le Plan du Palais de la Porte de Zelkova est dessiné sur un papier quadrillé, dont la plus petite unité carrée équivaut à la longueur d'une travée, un ken 間, soit approximativement deux mètres d'entre-axe des poteaux porteurs, pour une longueur de tatami autour de 1,80 m, une dimension variable de quelques centimètres selon de la largeur des poteaux. Le plan montre les travaux que le nouveau seigneur s'apprêtait sans doute à réaliser pour moderniser sa résidence secondaire. Il s'agit d'un document technique, en couleur, sur lequel sont portés les directions cardinales, les palissades et les portes (en rouge), le mur d'enceinte (en brun) et les édifices anciens (en bleu). Les bâtiments nouveaux (ou projetés) sont indiqués sur des papiers collés sur le plan, harigami 貼紙, bruns, orange et blancs. Le nom de la plupart des pavillons et de leurs salles sont calligraphiés sur le document, ce qui permet d'en reconstituer l'organisation avec précision.

Un corps d'édifice tout en longueur, nagaya 長屋, bordait la face sud-ouest, le long de la douve du château. Au centre, le porche d'entrée, gomon 御門, de trois travées de large, était en bois de keyaki 梘, l'orme du Japon (Zelcova serrata Thumb.), d'où le nom de "palais de Zelkova ». De part et d'autre du porche, étaient aménagés un poste de garde, gobanjo 御番所, et un banc d'attente, koshikake 腰掛, puis, sur le côté ouest, une salle des petits fonctionnaires, shōri 小吏, un garage à chaises

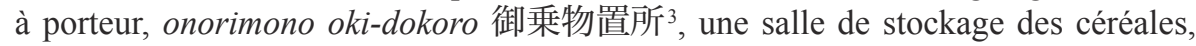
otsukiya 御春屋, dont la petite porte par laquelle on livrait du riz était protégée d'un dispositif «pour empêcher les souris d'entrer», nezumi fuiri 鼠不入. À l'extrémité ouest de l'édifice, un second poste de garde permettait de surveiller l'extérieur de toute la façade. Au nord de ce dernier, au milieu d'une cour, un édifice indépendant abritait les fourneaux, okamaya 御釜屋. À l'est du porche d'entrée, le poste de garde était contigu à une remise à outillage, kodōgu-beya 小道具部屋, puis à une salle de l'employé en charge des sandales du seigneur, zōri tori-beya 草履取部屋 ${ }^{4}$, et à celle du stockage du charbon de bois, sumi oki-dokoro 炭置所, que l'on utilisait pour les chaufferettes et braséros du palais. Quatre salles du personnel, équipées de latrines séparées, étaient aménagées à l'extrémité est de ce corps d'édifice.

2. Le document est référencé sous le no 44633 aux archives du musée du château de Hikone.

3. Norimono est un terme courant à l'époque d'Edo pour désigner les chaises à porteur, kago 駕籠.

4. Cet employé avait la charge de mettre et de retirer les sandales et les socques du seigneur lors de ses déplacements à l'extérieur du palais. 


\section{Le pavillon de l'entrée, le pavillon des fonctionnaires et de la cuisine}

Une fois passé le porche et traversé la cour, l'accès au palais se faisait par l'entrée principale, genkan 玄関, dont l'ouverture était décalée d'une travée et demie par rapport à l'axe du porche, ce qui imposait une progression en oblique. L'accès à l'entrée se faisait par un planché surélevé, shikidai 式台, de deux travées sur une, qui permettait d'atteindre la pièce d'entrée, de deux travées de large sur trois. Côté est, cette pièce donnait sur une salle de six tatamis de surface, elle-même contiguë à une autre, au plan carré, de quatre tatamis et demi : la salle des secrétaires (goyūhitsu no mima 御右 [祐] 筆御間) en charge de la prise des notes dictées par le seigneur. Ces deux pièces donnaient sur une vaste salle de service, goyō-beya 御用部屋.

En progressant depuis l'entrée vers le nord-est, on accédait à une petite pièce de quatre tatamis, elle-même contiguë à une salle de dix tatamis, tout en longueur, qui encadrait le vaste espace en terre battue, doma 土間, de l'entrée de service. De part et d'autre de l'entrée de service, se trouvaient la salle des petits employés, koyakuninbeya 小役人部屋, de six tatamis, et une seconde salle de service, goyō-beya, de sept tatamis et demi. L'espace de terre battue, qui jouxtait la salle des petits employés, séparait ainsi l'entrée principale de la cuisine, odai-dokoro 御台所.

La cuisine était une vaste salle carrée, équipée d'un foyer, irori 囲炉裏, et de fourneaux, kamado 窵, aménagés en son centre. Elle donnait, côté cour, sur un plancher au centre duquel se trouvait un puits. Le dessin laisse à penser que le plancher était fait de bambous, afin de permettre l'écoulement de l'eau. Un réseau enterré d'alimentation en eau traversait la cour intérieure et alimentait trois puits : celui du plancher et deux autres aménagés de part et d'autre de la cuisine.

Au nord de la cuisine, étaient alignées les salles du personnel de maison : salle des femmes de ménage, osue no ma 御末之間, avec un foyer aménagé en son centre, chambre des cuisiniers, (on) makanai-beya (御) 賄部屋, office des employés, goyōeki tsume-sho 御用役詰所, ainsi qu'une pièce des remèdes infusés, (o) yakusen-beya (御) 薬煎部屋. Puis venaient les 《salles de la chaîne», gojo-mae 御鎖前 et $u c h i$ gojō-mae 内御鎖前, où des gardes surveillaient les allées-et-venues des personnes et des biens entre la résidence privée et les autres espaces du palais, marquant ainsi la limite entre la partie officielle de la résidence, omote muki 表向, et les espaces privés du seigneur, oku-gata muki 奥方向.

\section{Les pavillons du service féminin}

Les femmes, joch $\bar{u}$ 女中, qui servaient au palais étaient divisées en deux catégories : les unes, de milieu paysan, formaient le personnel des servantes et des femmes de ménage; les autres, issues de familles de vassaux, étaient domestiques ou dames de compagnie, en fonction d'une hiérarchie de rang qui dépendait du rang vassalique de leur famille. Les sources mentionnent la naissance d'enfants parmi les femmes servant aux appartements privés, ce qui témoigne du fait que le seigneur entretenait des relations intimes avec certaines d'entre-elles.

Parmi le personnel composé de filles de paysans, l'osan no ma 御三之間, était une servante d'antichambre. Placée en haut de l'échelle des servantes, elle s'occupait du rangement, de l'approvisionnement en eau et de l'entretien des braséros du personnel féminin issu de famille de guerrier. Elle était aidée dans son travail par la petite 
servante d'antichambre, osan no ma kodomo 御三之間子供, dont l'âge, comme celui des autres petites filles travaillant au palais, allait de sept à quatorze ans. L'onakai 御中居 servait à la salle de préparation des repas, gozen-dokoro 御瞨所. Son rôle était important, car elle surveillait la préparation des mets en fonction du menu, comme la cuisson du poisson ou les dimensions des portions de pâte de soja, $t \bar{o} f u$ 豆, ou de radis blanc, daikon 大根 5 . L'ohi no ban お日の番, la « veilleuse des feux », avait pour mission de passer dans toutes les pièces pour y surveiller les lampes à huile, les braséros et les boîtes à tabac, afin d'éviter tout risque d'incendie. L'osue-gashira 御末頭 assumait la fonction de « cheffe des femmes de ménage », et dirigeait les osue et ohashita. L'osue 御末, la «femme de ménage », nettoyait quotidiennement la salle de bain et la salle de préparation des repas, ou allait chercher le riz dans la réserve pour le laver; elle était assistée de l'osue kodomo 御末子供. À l'ohashita 御半下, la femme de ménage de la plus basse condition, revenaient les tâches les plus viles.

Parmi les filles de vassaux, jijo 侍女, l'okoshō 御小姓 était une demoiselle d'honneur, âgée de sept à quatorze ans, en charge de petites tâches, comme l'approvisionnement des boîtes à tabac ou de l'eau de la toilette. Après quatorze ans, la demoiselle d'honneur devenait une dame de compagnie, otsugi 御次, et servait au palais comme domestique. Une épouse secondaire pouvait être choisie parmi les dames de compagnie, raison pour laquelle l'origine guerrière de sa famille et la beauté de ses traits étaient des éléments importants du choix d'une okoshō. L'otsugi avait aussi la charge des ustensiles précieux, comme ceux du culte bouddhique, de la cérémonie du thé ou de la calligraphie. Elle tirait son nom du tsugi no ma 次の間, la « salle attenante » [à la pièce principale du seigneur], où elle devait se tenir toujours prête à intervenir pour servir son maître. Quant à la gonando 御納戸, elle était en charge de la garde-robe du Seigneur.

Au nord de la cuisine, deux pavillons intermédiaires (en orange et en gris sur le plan) pour le personnel féminin conduisaient aux appartements privés de Monseigneur, ooku goten 御奥御殿, le « pavillon du Fond ». Le premier était équipé de deux salles des demoiselles d'honneur, okoshō-beya 御小姓部屋, une salle de Thé, ocha no $m a$ 御茶之間, et une nouvelle extension pour le personnel de service (en orange sur le plan), avec une chambre des Préposées à la préparation des repas, nakai-beya 中 居部屋, une chambre des dames de compagnie, otsugi-beya 御次部屋, une chambre des servantes des dames de compagnies, otsugi geshin 御次下志ん, les chambres des kamijin 上志んet des shimojin 下志ん, et trois latrines.

Au nord de ce bâtiment, le pavillon jouxtant l'édifice privé de Monseigneur comprenait une salle de l'étagère daisu, odaisu no ma 御台子之間, où l'on faisait chauffer l'eau et préparait le thé pour le seigneur - une pièce de huit tatamis. Cette pièce, comme la domestique qui en avait la charge, tirait son nom de l'étagère utilisée dans les cérémonies du thé formelles. Les servantes d'antichambre, osan no ma 御三之間, occupaient une salle de dix tatamis, contigüe à une salle des secrétaires, goyūhitsu no ma 御祐筆之間, d'une superficie de treize tatamis.

5. Par cette fonction, l'onakai avait été affublée au palais d'Edo du sobriquet de la « dorade », otai お鯛, ou de celui du « poulpe», otako お蛸. 


\section{Le pavillon des appartements privés de Monseigneur}

L'extrémité nord-ouest de cette enfilade d'édifices était occupée par le pavillon des appartements privés, dont la pièce principale de dix tatamis était la salle de Monseigneur, goza no ma 御座之間 (litt. « salle du siège »), disposée dans l'angle nord-est de l'édifice, et ornée d'un autel d'ornement, toko no ma 床の間, et d'étagères décoratives, kazari-dana 飾棚. Au sud de celle-ci, l'otsugi se tenait dans la salle attenante, de dix tatamis de surface. La salle de Monseigneur jouxtait à l'ouest une salle de huit tatamis et demi, elle-même contigüe (côté sud) à la chambre à coucher, gyoshin no ma 御寝之間, de dix tatamis de surface. Une salle de treize tatamis et demi équipée d'un placard, oshiire 押入, servait de garde-robe, gonando 御納戸. Le coin nord-ouest du pavillon était occupée par la petite salle de l'autel bouddhique, butsuma 佛間. À proximité, dans le coin sud-ouest, se trouvait l'édifice du bain, oyu-dono 御湯殿, qui comprenait la salle de bain, un vestiaire où le seigneur se changeait, une salle des domestiques et une latrine. Le pavillon de Monseigneur était longé du côté des pièces nobles par une coursive, engawa 縁側, d'une travée de large, elle-même bordée d'une second plancher d'une demie travée.

\section{Le pavillon de détente et le terrain de jeu de balle au pied}

À l'extrémité nord-ouest du pavillon de Monseigneur, un couloir de trois travées de long menait à un pavillon de plaisance, ochin お亭, à un étage, d'où l'on pouvait contempler la vue sur le lac Matsubara. La pièce principale de ce pavillon, de dix tatamis de surface, était équipée d'un autel d'ornement et bordée d'une coursive en tatamis d'une travée de large. Une pièce secondaire d'une superficie équivalente complétait le premier niveau. Le plan mentionne une latrine et une pièce d'eau, nouvellement aménagées. On retrouve le dessin de ce pavillon sur tous les plans ultérieurs, jusqu'au XIX ${ }^{\mathrm{e}}$ siècle, de même que sur les peintures du parc Genkyūen, où il est alors le nommé le «Belvédère du Plaisir Tranquille», Anrakutei 安楽亭. L'édifice était relié au pavillon de Monseigneur par un couloir de liaison, rōka 廊下. En vis-à-vis de ce belvédère, au centre d'une cour, un terrain de jeu, mariba 鞠場, avait été projeté, afin d'y pratiquer le kemari 蹴鞠, un antique jeu de balle au pied à nouveau en vogue dans le Japon du XVIII' siècle $^{6}$.

\section{La partie officielle du palais}

Les corps d'édifice bâtis sur le côté est de la cour centrale du palais de Zelkova abritaient la partie officielle de la résidence, où le seigneur recevait des invités. Les différents documents montrent que cette partie du palais a été réaménagée à plusieurs reprises. Tout porte à croire que les indications portées sur le plan présentent la chronologie suivante : en bleu sombre, l'état initial de la première période, en bleu clair l'adjonction du pavillon de la salle de Monseigneur, en jaune pâle la modification des pièces de ce pavillon et, en orange, un réaménagement plus important de cette partie du palais. Les parties en bleu clair dessinent des esquisses, non réalisées de divers projets d'aménagement.

6. Le plan montre que le terrain de jeu était alors en cours de réaménagement, car un dessin plus ancien recouvert d'un papier collé indique qu'il avait été implanté plus au sud. 
Selon ces indications, l'édifice à l'usage du personnel (en orange) présente la disposition intérieure des pièces : salle de l'Intendant, gonando-beya 御納戸部屋, en charge de la garde-robe et du mobilier, salle de la garde-robe et des mobiliers, gonando 御納戸, et chambre des chambellans, osobayaku-beya 御側役部屋.

Le principal pavillon (en bleu clair) abritait la salle de Monseigneur, goza no ma. Cette salle mesurait douze tatamis de surface et était équipée d'un autel d'ornement, d'étagères décoratives et d'un bureau encastré, tsuke shoin 付書院. Elle donnait à l'est sur une large coursive d'angle, d'une travée de large, que bordait d'un plancher étroit, sunoko 筫の子. Au sud de la salle de Monseigneur, une salle attenante de dixhuit tatamis de surface a été transformée en scène de théâtre nō, raison pour laquelle elle apparaît coloriée en jaune sur le document. Le plan porte la mention «oshikibutai 御敷舞台 », un terme qui désigne une scène provisoire, aménagée à l'intérieur d'un édifice quelconque, et qui la distingue d'une scène permanente, honbutai 本舞 台. Par cet aménagement, qui consistait à recouvrir le plancher de la salle attenante par un second plancher surélevé pour la scène de nō, il était ainsi possible d'organiser des représentations théâtrales et de danse à l'intérieur même du pavillon. Autour de la scène, on avaient aménagé les équipements utiles à une représentation : le «pont», hashi-gakari 橋掛, par lequel entraient et sortaient les acteurs, le siège arrière, ato-za 後座, où prenait place l'orchestre, hayashi 囃子, la place du deutéragoniste, waki-za 脇座, et la salle du Miroir, kagami no ma 鏡の間, où les acteurs changeaient de tenue et ajustaient leur masque ${ }^{7}$. Dans ce plan, la scène carrée mesurait trois travées carrées et le pont trois travées et demie de long. À l'arrière de la scène, le plan porte la mention «hame はめ», ce qui désigne des planches encastrées les unes dans les autres, dites hameita 羽目板; il s'agissait là, sans doute, du « panneau du miroir », kagamiita 鏡板, la paroi en bois de cyprès orné d'un décor de vieux pin, oimatsu 老松8. Sur le côté, un passage pour entrer et sortir, deiri-guchi 出入口, remplaçait à la «porte de séparation », kirido-guchi 切戸口, aménagée usuellement dans l'angle arrière-droit d'une scène de nō pour l'entrée des musiciens.

La face sud-est du pavillon donnait sur un jardin clos d'une palissade, que le plan ne représente pas et dont le motif paysager reste inconnu. La face ouest du pavillon des réceptions était utilisée pour le service, comme l'indique les parties en orange et en bleu dessinées sous une couche de papiers bleu ciel, où l'on découvre deux bains et un couloir de liaison transversal permettant de rejoindre les appartements privés du seigneur.

7. Voir la description d'une scène véritable, honbutai, dans Nicolas Fiévé, « Le château des seigneurs Ii de Hikone, ses palais et ses jardins à l'époque d'Edo. Deuxième partie : l'organisation spatiale du palais seigneurial », Annuaire. Résumé des conférences et travaux, 151ªnnée, 2018-2019, Paris, EPHE, PSL, SHP, 2020.

8. Le thème du vieux pin toujours vert évoque la longévité, et dans le théâtre nō le motif est une ode à la vertu impériale (dont le vieux pin est un symbole), gardienne entre toute de la poésie de waka, comme l'expriment tant de pièces du répertoire. 


\section{Les transformations du palais de Zelkova, au milieu de la seconde moitié du XVIII siècle}

Le «Dessin complet du palais de Zelkova », Keyaki goten subete egaki no zu 槻御殿總画図 ${ }^{9}(121,5 \mathrm{~cm}$ par 147,5 cm) est un document non daté. Comparé au plan de 1754, il montre plusieurs évolutions importantes du dispositif spatial, changements qui apparaissent sur le document coloriés en jaune. La première concerne l'extension de la résidence sur le côté est avec la construction d'un palais officiel (oomote), désormais indépendant de la résidence privée (ooku-gata). Un mur sépare nettement les deux zones spatiales. L'extension a nécessité un réaménagement du porche d'entrée, du pavillon de l'entrée et l'adjonction de plusieurs salles de service. Enfin, la résidence privée est elle-même agrémentée de deux nouveaux pavillons, implantés dans la cour centrale, dont les dessins avaient été esquissés sur plan précédent à l'aide de papiers collés de couleur bleu pâle. Parmi les évolutions du plan, on trouve aussi l'aménagement d'une porte de la chaîne au-dessus du pavillon de l'entrée, ce qui marque un déplacement de la séparation entre la partie privée et la partie officielle du palais. Cette porte de la chaîne, gojo-guchi 御鎖口, est une pièce de seize tatamis contrôlée par un poste de garde.

À l'arrière des cuisines, le pavillon des servantes est demeuré inchangé depuis le plan de 1754, de même que celui du personnel féminin, bien que les noms des pièces aient été modifiés. On y trouve, dans l'ordre de progression vers le pavillon de Monseigneur, une salle de préparation pour les domestiques, jochū shitaku-dokoro 女中 支度所 (de huit tatamis), contiguë à une salle de thé, ocha no ma (de douze tatamis), équipée d'un grand foyer irori 囲炉裏 et de deux placards de rangement, et une antichambre, tamari no ma 溜之間 (de huit tatamis). Sur le côté ouest de l'édifice, ont été aménagés un bain et une latrine pour le personnel. Le long du couloir de liaison, rōka, vient ensuite la salle des domestiques, otsugi (de huit tatamis), celle des servantes d'antichambre, gosan no ma, la salle de l'étagère daisu, odaisu no ma (de huit tatamis), où l'on prépare le thé du seigneur, celle des secrétaires, goyūhitsu no ma (de treize tatamis), et une latrine. Au nord du pavillon du service féminin, ni le pavillon de Monseigneur, goza no ma mune 御座之間棟, ni le pavillon à étage, ochin, n'ont été modifié. Les autres parties notées en jaune sur le document représentent les esquisses d'un projet d'agrandissement du palais, que l'on découvre avec précision sur le Plan de la résidence [bâtie] à l'extérieur de la Porte Noire (voir infra, p. 491-492).

\section{Les transformations du palais de Zelkova à la fin du XVIII' siècle}

Le «Plan de la résidence [bâtie] à l'extérieur de la Porte Noire», Kuro-gomon soto oyashiki oezu 黒御門外御屋敷御絵図 ${ }^{10}$, est un document non daté, mais paraphé par l'administrateur secondaire en charge de la construction des nouveaux bâtiments, osakuji kata 御作事方 ${ }^{11}$. Il s'agit d'un document officiel sur l'implantation

9. Le document est référencé sous le n 44858 aux archives du musée du château de Hikone. Le plan est aussi répertorié dans plusieurs documents sous l'intitulé «Plan de la résidence [bâtie] devant la Porte noire », Kuro gomon mae yashiki ezu 黒御門前屋敷絵図.

10. Le document est référencé sous le $\mathrm{n}^{\circ} 44630$ aux archives du musée du château de Hikone.

11. Le sakuji kata ou sakuji bugyō 作事奉行 a été officialisée par le bakufu en 1632 (Kan.ei 9), bien qu'elle existait dans les faits depuis le début de l'époque d'Edo. Voir Nicolas Fiévé, « Le monde de la charpen- 
des pavillons et des salles du palais de Zelkova réalisé au cours de la seconde moitié du XVIII ${ }^{e}$ siècle, et sur le projet d'agrandissement de la partie officielle de ce palais. Il montre le dessin sommaire d'un jardin, pour la première fois représenté sur un plan du palais, avec un grand plan d'eau, des allées et un pont. Les édifices sont dessinés à échelle juste, à l'encre de Chine noire, et des ajouts de papiers collés indiquent par aplat en jaune le projet des nouvelles extensions.

Le document témoigne d'une volonté de réaménagement en profondeur du palais. Dans ce nouveau plan, le porche d'Entrée ne se situe plus au centre du long bâtiment d'enceinte de la face sud-ouest, mais à l'est du palais, où il donne désormais sur une vaste cour d'entrée. Il est ceint de part et d'autre d'une salle au sol en terre battue et d'un poste de garde.

À l'emplacement de l'ancien porche, au centre du long bâtiment, a été aménagé une vaste cuisine, dont le dessin recouvert d'un papier blanc laisse à penser qu'il s'agit d'un projet. À l'ouest de la cuisine, se suivent respectivement une remise, okimono, une salle de stockage des céréales, otsukiya, une réserve à riz, kome-gura 米蔵, une chambre pour les produits salés, shiomasu-beya 塩増部屋, puis une réserve à fourrage, kaiba okimono 粥葉置物, une chambre de palefrenier, umatori-beya 馬取部屋, une écurie à trois box et un poste de garde. Sur l'aile est, on trouve un autre poste de garde, une salle pour le stockage du charbon de bois, une réserve de bois de chauffage, takigi-beya 薪部屋, une salle à chaises à porteur, okago-beya 御駕部屋, une salle de surveillants, metsuke 目付, une remise, okimono, une remise pour les ustensiles à cérémonie du thé, sado okimono 茶道置物, une salle des moines servant au palais, $b \bar{o} z u$ 坊主, une salle d'exposition des arcs, yumi goranjō 弓御上覧場, et une salle de garde au sol en terre battue, doba ban-dokoro 土場番所.

Le pavillon de l'Entrée a conservé un dispositif semblable à celui du plan ancien, bien que la répartition de ses salles ait été modifiée ici et là, de même que l'entrée de service au sol en terre battue, l'ancienne cuisine et la salle indépendante des fourneaux.

\section{La partie officielle du palais}

Le Kuro-gomon soto oyashiki oezu montre deux états du plan de la partie officielle du palais. Un nouveau corps de bâtiment de la partie officielle du palais a été ajouté à l'est du pavillon de l'entrée. Le dessin de cet édifice, initialement colorié en jaune, a été recouvert d'un papier blanc collé sur le plan, papier sur lequel est indiquée une nouvelle extension, dont la construction effective est confirmée par les documents plus tardifs (voir infra, p. 494).

Selon le dessin initial, qui montre l'état de la partie officielle du palais au cours de la seconde moitié du XVIII ${ }^{\mathrm{e}}$ siècle, ce long bâtiment disposé nord-est / sud-ouest était composé d'une enfilade de pièces, où l'on trouvait, du sud-ouest vers le nord-est : une remise, une salle du siège, goza no ma, une salle attenante, otsugi no ma, une antichambre, san no mima, puis une seconde salle attenante et une seconde salle du siège.

Un dessin ajouté sur une bande de papier indique un déplacement de l'édifice principal, décalé d'une vingtaine de mètres vers le sud-est, afin d'agrandir la cour pour y bâtir les nouveaux pavillons de la résidence privée. La principale modification

terie et les reconstructions du Palais impérial, dans le Kyōto du XVII ${ }^{\mathrm{e}}$ siècle », Annuaire. Résumé des conférences et travaux, $148^{e}$ année, 2015-2016, Paris, EPHE, PSL, SHP, 2017, p. 388-395. 
concerne l'ajout d'un nouveau pavillon de réception, shoin 書院, équipé d'un niveau surélevé, jōdan 上段, aménagé dans le coin est du palais et donnant sur un jardin. Dans ce nouvel aménagement, le pavillon des appartements privés est directement relié par un couloir de liaison aux espaces officiels. Le même couloir permet de rejoindre deux salles de bain, installées entre les pavillons des parties privée et officielle de la demeure. Le jardin qui fait face au nouveau pavillon est une extension du parc Genkyūen 玄宮園, le grand jardin promenade de trois hectares aménagé sur les bords du lac Matsubara. Des chemins de sable fin, kosuna 小砂, font le tour du plan d'eau, eux-mêmes bordés des pelouses, siba 芝. Un pont enjambe la pièce d'eau à l'endroit où celle-ci débouche sur une petite vallée, tani 谷, artificielle.

\section{La partie privée du palais}

À l'ouest du palais, la résidence privée a été entièrement transformée. Un premier corps de bâtiment abrite une chambre des préposées à la préparation des repas, onakai-beya, une chambre des otsugi-shimojin 御次下陣, ainsi que les bains du personnel, sōyu-dono 惣湯殿. Au nord de cet édifice, un nouveau corps de logis comprend quatre chambres identiques pour les dames d'honneur, de huit tatamis de surface et équipées chacune de trois placards, oshiire 押し入れ. Entre ces deux corps de logis, l'ancienne salle pour le thé, ocha no ma, équipée d'un foyer, est devenue une salle des femmes de ménage, osue no ma. Elle jouxte la salle de préparation des employées, jochū shitaku-dokoro 女中支度所 (de 8 tatamis), et une antichambre, tamari no ma. Plus au nord, mais désormais indépendant du reste des édifices, le pavillon à étage, ochin, est demeuré inchangé.

Si l'on compare ce document au plan de 1756, on constate que le pavillon du service féminin et celui de Monseigneur ont été déplacés pour être reconstruits au centre de la demeure. Le pavillon du service comprend quatre pièces : salle de l'étagère daisu, garde-robe, salle des servantes d'antichambre, salle des secrétaires. $\mathrm{Au}$ nord-est de ce pavillon est aménagé l'appartement privé, avec la salle de Monseigneur (goza no $\mathrm{ma}$ ), une salle de dix tatamis, ornée d'un autel d'ornement et d'étagères décoratives. Sur le côté sud-est de celle-ci, la salle attenante mesure dix tatamis de surface. Sous la salle de Monseigneur, une salle de huit tatamis et demi est attenante à la chambre à coucher, de dix tatamis et équipée d'un placard d'une demi-travée de large. La garde-robe est une salle de treize tatamis et demi avec un placard d'une travée de large. Contiguë à la chambre à coucher, la pièce de l'otsugi est équipée d'un placard d'une demi-travée. Les salles exposées au nord-est et au sud-est sont longées d'une coursive d'un tatami de large, équipée de volets que l'on range dans des coffres à volets. Sur la face nord-ouest, au-delà d'un couloir de service, on trouve des latrines et une pièce de rangement, okimono, équipée de placards.

\section{Le réaménagement du palais après 1812, à l'occasion de la retraite du seigneur Naonaka}

Le Plan de la résidence [bâtie] à l'extérieur de la Porte Noire présentait un projet du palais, que confirme le «Plan illustré du palais Keyaki », Keyaki goten ozu 梘御殿 御 $(127 \mathrm{~cm} \times 171,5 \mathrm{~cm})^{12}$, un document officiel signé de l'administrateur en charge de

12. Le document est référencé sous le $n^{\circ} 44631$ des archives du musée du château de Hikone. 
la construction. Précieux à bien des égards, ce plan daté de 1818 (Bunka 15) présente les réaménagements réalisés à partir de 1812 pour faire de la villa secondaire de la famille Ii la demeure principale du seigneur Naonaka. Ii Naonaka avait renoncé à ses fonctions officielles au bakufu cette année-là et était rentré l'année suivante à Hikone, accompagné de sa famille. Suite à ces travaux, le porche d'entrée et la salle d'entrée ont été déplacés, de même que le mur d'enceinte sud, afin de permettre l'aménagement d'une vaste cour d'entrée. L'édifice en longueur de la partie officielle du palais a été décalé vers le sud-est afin de bâtir une scène de nō dans la cour centrale, l'édifice de Monseigneur a été reconstruit et la demeure a été agrémentée de plusieurs pavillons de thé. Dans la partie privée de la résidence, de nouveaux pavillons ont été aménagés pour la famille de Naonaka, rentrée en partie elle aussi d'Edo à Hikone.

Alors que les documents précédents ne distinguaient pas les espaces officiels des appartements privés, la légende du plan montre la distinction faite entre les espaces du palais officiel (oomote) représentés en jaune et ceux du palais privé (ooku kata) en rose foncé. Un trait rose pâle indique les puits et les réservoirs d'eau, le brun les édifices au sol en terre battue, et des lignes roses dessinent les palissades en planches de bois, ita-bei 板塀, qui délimitent les différents espaces du palais.

\section{L'entrée principale}

Le plan de 1818 présente des espaces de réception du palais officiel entièrement modifiés et considérablement agrandis. Selon ce document, l'enceinte sud-ouest délimite une vaste cour, dans laquelle on pénétrait par un porche honneur, oomote-mon 御表門, aménagé dans le coin sud-est. Le porche donnait d'un côté sur un poste de garde, gobansho, et de l'autre sur un espace couvert équipé d'un banc d'attente. La cour d'honneur avait la forme d'un carré parfait, et il fallait la traverser pour accéder à l'entrée, genkan, dont l'ouverture était orientée vers l'est, la direction faste du levant. Deux degrés permettaient d'accéder à une pièce de quinze tatamis de surface équipée d'un autel d'ornement. De là, un virage à quatre-vingt-dix degrés permettait d'accéder à une pièce de quatorze tatamis de surface qui menait vers le nord-est au palais officiel.

\section{Les salles du personnel et les cuisines}

Une porte de service, adjointe d'un poste de garde, conduisait aux cuisines, aménagées à l'ouest du mur de façade. Dans ce nouveau plan, l'ancien long bâtiment de façade a été remplacé par un édifice à l'usage du personnel : secrétaires, goyūhitsu 御右筆, surveillants, metsuke 目付, hommes de service, goyōnin 御用人, chambellans, osobayaku 御側役, pages, okoshō 御小姓, bonze des appartements privés, oku gobōzu 奥御坊主. On trouve aussi une pièce pour le thé, ochaya 御茶屋, une salle de la pendule, tokei 時計, une autre du surveillant des plateaux, gozenban 御膳番, un placard de rangement, monooki 物置, l'office des communs, onmakanai tsume-sho 御 賄詰所, le service de la table seigneuriale (où l'on préparait les plateaux), gozen kata okimono 御膳方置物, la cuisine, dai-dokoro, la salle des plateaux, gozen-beya 御膳 部屋, la salle du chef de cuisine, oita-gashira 御板頭, la salle des premiers commis, onmakanai tedai 御賄手代.

Le document montre que le projet de construction d'une nouvelle cuisine esquissé sur le Plan de la résidence [bâtie] à l'extérieur de la Porte Noire a bien été réalisé (voir supra, p. 491). Dans le nouveau plan, le dispositif du bâtiment des fourneaux, 
kamaya 釜屋, est demeuré inchangé, isolée au milieu de la cour afin de limiter les risques d'incendies. La salle des fourneaux est contiguë à un bain pour le personnel de cuisine. Le long du couloir se trouve une remise à lanternes, andon oki-dokoro 行燈置所.

Dans la partie centrale, l'entrée de service en terre battue est conservée, mais l'espace de l'ancienne cuisine a été réaménagé en un ensemble de salles pour le personnel : un bureau du régisseur, motoshime-dokoro 元 $邓$ 所, une pièce des employés subalternes, kozukai idoko 小使居所, un poste de garde de la porte de la chaîne, gojō-mae ban-dokoro 御鎖前番所, une salle des vêtements, gofuku no ma 只服之 間 pour la fabrication et l'entretien des vêtements, un poste supérieur de garde de la porte de la chaîne, gojō-mae kami-ban-dokoro 御鎖前上番所, une porte de la chaîne », gojō-guchi 御鎖口, une salle d'accueil, shukuhō no ma 宿逢之間, une salle des employés et des domestiques, goyōshi gokushi-yaku 御用使御櫛役, et un bureau du médecin, goishi 御医師.

\section{Les salles de réception du palais officiel}

Le déplacement plus à l'est du corps d'édifice du palais officiel a permis de dégager une cour spacieuse, afin d'y installer une scène de nō permanente. Dans le nouveau dispositif, le pont d'accès à la scène, hashi-gakari, conduit à la salle du miroir où se préparent les acteurs. Du nord vers le sud, le corps d'édifice du palais officiel comprend une salle du siège, goza no ma, de huit tatamis et équipée d'un autel d'ornement, une salle attenante de dix tatamis et une antichambre de huit tatamis. Les trois pièces servaient de loge à l'occasion d'une représentation théâtrale. Dans le prolongement de ces salles, une pièce intermédiaire donnait sur deux pièces de rangement : un petit cabinet des effets précieux, gokonando monooki 御小納戸物置, et un guichet de l'intendant du petit cabinet, gokokonando tsume-guchi 御小納戸詰口.

À l'est de ce corps d'édifice, une vaste cour abritait la salle d'escrime, keiko koya 稽古小屋 (on disait aussi keiko-dokoro 稽古所) et une cible pour l'entrainement au tir à l'arc, placée sur un monticule de terre, azuchi 垛. Plus à l'est encore, deux courettes abritaient des réserves, deux pour le palais officiel, dont l'une à compartiments, shikiri dozō 仕切土蔵, et une autre pour les mobiliers du petit cabinet aux effets précieux, konando dozō 小納戸土蔵, ainsi que deux autres pour les mobiliers destinés aux appartements privés du seigneur, okugata dozō 奥方土蔵.

\section{Le palais privé}

Au nord-ouest du palais, l'édifice des chambres du personnel a été remplacé par trois nouveaux corps de logis pour les dames de compagnie, les domestiques et les servantes. Chacun de ces édifices est équipé de foyers pour chauffer l'eau et de quatre latrines. Un vaste bain commun, sōyu-dono 惣湯殿, est installé à proximité.

Accolé aux corps d'édifice des domestiques et des dames de compagnie, l'office des communs comprenait une salle de la préposée aux plateaux, gozen-kata 御膳方, une chambre des plateaux, gozen-beya 御膳部屋 et une pièce pour ranger les lanternes, andon oki-dokoro 行燈置所. À l'est de l'office, l'édifice de jour où se tenaient les dames de compagnie comprenait cinq pièces : la salle de l'étagère daisu, la salle du thé (ocha no ma), la garde-robe (gofuku no ma), la salle des servantes d'antichambre et la salle des secrétaires. 
À l'est de l'édifice des dames de compagnie, un petit pavillon était réservé à Tetsusaburō 鉄三郎, le fils héritier de Naonaka (le futur $13^{\mathrm{e}}$ seigneur Naosuke 直柘), né dans ce palais en 1815. Le pavillon comprenait une salle du siège, goza no ma, et une salle attenante pour l'otsugi. Le pavillon du fils héritier était séparé de la partie officielle de la demeure par la salle de l'autel bouddhique, butsudan no ma, et par deux salles de bains, oyu-dono. Trois autres pavillons ont été ajoutés au nord-ouest de la résidence privée, afin d'accueillir les autres membres de la famille de Naonaka. Chacun de ces pavillons était composé d'une salle du siège, d'une salle attenante et d'une vaste coursive et donnait sur un jardinet privatif.

Naonaka dormait au centre de la résidence privée, dans un pavillon où se trouvait la chambre à coucher, adjointe d'une salle attenante et d'une salle de la garderobe, gonando no ma. Sur le côté sud de ce pavillon, au-delà d'un couloir, avaient été aménagées les bureaux des dames de compagnie et des domestiques, otsugi joch $\bar{u}$ tsumesho 御次女中詰所 et osoba jochu tsumesho 御側女中詰所.

Les deux principaux édifices des appartements privés étaient le pavillon de Monseigneur, goza no ma mune 御座之間棟, et le pavillon du Salon du Fond, oku goza no ma mune 奥御座之間棟, pour son épouse. De ces deux pavillons nouvellement bâtis pour Naonaka a subsisté le pavillon de Monseigneur, qui est désigné dans les textes tantôt par onshin-dachi 御新建, « la nouvelle construction », tantôt par shinshoin 新書 院, le « nouveau shoin ». Ce pavillon comprenait une salle du niveau supérieur, jōdan no ma 上段之間, de dix tatamis de surface, avec un autel d'ornement, des étagères décoratives et un bureau décoratif; une salle de séjour haute, kami no ima 上之居間, de douze tatamis de surface, une salle attenante et un petit salon, okozashiki 御小座敷, un dispositif inchangé dans l'actuel pavillon. Depuis son pavillon, le seigneur pouvait accéder directement aux salons des espaces officiels du palais, mais il devait pour cela traverser la pièce du grelot, osuzu no ma 御鈴之間 et le poste de garde de la porte de la chaîne, gojō-guchi.

Sur la face nord du pavillon de Monseigneur, un édifice porte la mention «tono sama 殿様 », le « Maître ». Ce salon privé de Naonaka comprenait une salle du siège, de huit tatamis de surface avec un autel d'ornement d'une travée, une salle attenante, de sept tatamis et demi et une antichambre, de neuf tatamis et demi, au sud de laquelle un appentis abritait une pièce de quatre tatamis et demi, nommée ocha-dokoro 御 茶所, 《 espace pour le thé ». Cette salle pour préparer le thé jouxtait une latrine à l'usage de Naonaka.

Depuis le salon privé, vers le nord, Naonaka pouvait accéder directement à un petit bureau, naka zashiki 中座敷, donnant sur le jardin, alors que depuis l'antichambre, le personnel pouvait accéder à une remise, okimono. Ces deux pièces, le petit bureau et la remise permettaient de rejoindre une maison de thé, nouvellement construite à l'extrémité nord-est de la résidence et notée sur le plan ocha zashiki 御 茶座敷, 《salon pour le thé ». Ce pavillon, communément appelé Jishin no ma 地震 の間, « la chambre des Tremblements de terre», servait de refuge en cas de séisme. La pièce principale couvrait huit tatamis de surface et était équipée d'un autel d'ornement, d'une coursive ouverte sur le jardin et d'un niveau surélevé, jōdan no ma, de deux tatamis et demi, orné d'un autel d'ornement. Un dispositif spatial qui n'a pas 
changé depuis lors, cette maison de thé étant un des rares édifices de l'ancien palais encore conservés de nos jours.

À l'occasion de l'extension du palais pour Naonaka, le jardin fut entièrement réaménagé, comme en témoignent les vestiges actuels. Le Plan illustré des jardins du palais de Keyaki, Keyaki goten niwa ezu 槻御殿庭絵圖 ${ }^{13}$, montre le dispositif d'un jardin en style de pièce d'eau et de source, chisenshiki teien 池泉式庭園, c'est-à-dire organisé autour d'un étang agrémenté d'îles, qu'alimentait une source en forme de chute d'eau.

\section{Les maisons de thé au XIX $X^{\mathrm{e}}$ siècle}

Le «Plan de la résidence [bâtie] devant la Porte Noire», Kuro-gomon mae oyashiki ezu 黒御門前御屋敷絵圖 ${ }^{14}$, montre l'adjonction de pavillons de thé au cours de la première moitié du XIX ${ }^{\mathrm{e}}$ siècle, à l'époque du $12^{\mathrm{e}}$ seigneur Naoaki et du $13^{\mathrm{e}}$ seigneur Naosuke 井伊直矶 (1815-1860), deux de fervents adeptes des réunions de thé.

D'après ce plan, le petit bureau au nord du salon privé a été remplacé par un pavillon de thé indépendant, osukiya 御数寄屋, bâti au milieu d'un jardin. Au nord de la chambre du Tremblement de terre, un salon intermédiaire, naka zashiki, a été ajouté et équipé d'un autel d'ornement et d'étagères décoratives, de même qu'une pièce pour les servantes, sue. Un nouveau pavillon, Rakuraku no ma 楽々之間, la « salle des Félicités », complète ce groupe d'édifices de plaisance. Alors que les autres salles de thé étaient destinées à l'art du thé en poudre, matcha 抹茶, la salle des Félicités est un pavillon conçu pour l'art du thé en feuilles sencha, 煎茶, une pratique en vogue parmi les élites intellectuelles de la période.

Lorsque le palais fut détruit après la restauration de Meiji, le pavillon de Monseigneur et les pavillons de thé furent conservés. La famille Ii loua alors la propriété qui devint une auberge, et c'est à cette époque qu'il fut a ajouté une entrée d'honneur, au sud du pavillon de Monseigneur, le long de l'ancienne porte de la Chaîne. On construisit aussi un nouveau salon, shin-yashiki 新屋敷, et plusieurs bâtiments de service (cuisines, bains, toilettes, logements de fonction), des équipements sans valeur patrimoniale qui, hormis l'entrée d'honneur, ont aujourd'hui disparu.

Trois nouveaux pavillons furent aménagés dans le prolongement de la salle des Félicités : la chambre des Pins, Matsu no ma 松の間, la chambre des Grèbes castagnieux, nio no ma 鳰の間, et les Nouveaux salons de l'Est et de l'Ouest, Shin-nishi no ma et Shin-higashi no ma. Ces trois pavillons donnaient sur le paysage grandiose du lac Matsubara. Dans le nouveau dispositif de l'auberge, la maison de thé, osukiya, fut supprimée, et la remise, okimono, devint une « chambre des Moineaux », Suzume no ma 雀の間. Le Jishin no ma est demeuré inchangé, mais le salon intermédiaire a été divisé afin d'aménager deux nouvelles chambres, l'une dite des Feuilles d'automne, Kōyō no ma 紅葉之間, l'autre de la Neige, Yuki no ma 雪の間, une pièce de cinq tatamis et demi avec un autel d'ornement et des étagères décoratives. Quant à

13. Le document $(127,7 \mathrm{~cm} \times 176,2 \mathrm{~cm})$ est répertorié dans les archives du musée du château de Hikone sous le $n^{\circ} 61239$.

14. Le document, non daté, est répertorié dans les archives du musée du château de Hikone sous le $\mathrm{n}^{\circ} 61235$. 
l'ancienne salle des servantes, elle a été réaménagée elle aussi en chambre et nommée Okaru no ma おかるの間, sans doute du nom de l'héroïne célèbre de la pièce de théâtre Chūshingura 忠臣蔵.

\section{Les Jardins des courettes du palais de Zelkova à l'époque de Naonaka}

Les archives du musée du château de Hikone conservent quatre séries de dessins des jardinets de dix courettes, tsuboniwa 坪庭 ou nakaniwa 中庭, du palais de Zelkova ${ }^{15}$. Bien que ces dessins ne présentent pas une grande qualité de finition, ils sont d'une grande valeur pour l'historien, car il est extrêmement rare de connaître l'apparence des jardinets de courette d'un palais disparu. Les quatre séries de dessins présentent les mêmes aménagements paysagers; seule la qualité de rendu les différencie, avec une première série peinte à l'encre noire, alors que les trois autres sont en couleurs.

Le document marqué du caractère « $い$ » présente la cour aménagée devant la façade est du pavillon de réception du palais officiel, comme l'indique l'inscription omote goza no ma mae oniwa 表御座之間前御庭. Le dessin manque dans la première série, mais on le retrouve dans les trois autres. La cour comprend deux assemblages de minéraux et de pins : l'un est composé d'une imposante roche dressée, tateishi 立石, adjointe de deux pins aux troncs droits, plantés au bord de la coursive. Ce dispositif impose une verticalité qui s'oppose à l'horizontalité de la coursive. Le motif central du décor est un vieux pin noueux, taillé en forme de pin-parasol, karakasa matsu 傘松, planté sur une butte de terre ceinte de cinq roches et de rares végétaux.

Le nombre impair de cinq roches est conforme aux principes de disposition des pierres rapportés par les traités sur l'art des jardins de la période. Selon le principe d'un groupe de trois roches auquel on associe un second groupe de deux roches, un dispositif qui avait été développé depuis l'époque de Muromachi (1336-1573) dans les jardins de Kyōto, l'assemblage des pierres se faisait à partir d'une suite de nombres impairs $(3,5$ ou 7 , des nombres qui se divisent en sous-groupes : $3=2+1$; $5=3+2$ et $7=5+2$ ) et d'un placement en triangle des roches ou des groupes de roches. La disposition des pierres reposait sur le "rocher-maître », omo ishi 主石, auquel étaient adjointes deux roches de côté, waki ishi 脇石 : une roche d'ajout, soe ishi 添石, accolée au rocher-maître et une roche-invité, kyaku ishi 客石, placée légèrement à distance des deux premières. Dans ce dispositif, le rocher-maître et la roche d'ajout formaient une entité puissante qui attirait à elle la roche-invité, faisant naître ainsi la tension sur laquelle reposait la dynamique du paysage. Cet ordonnancement partait le plus souvent du fond du jardin, à gauche (de la vue sur le jardin depuis le bâtiment), pour aller vers la droite au premier plan du paysage. Si le nombre de trois roches passait à cinq - ce qui était le dispositif le plus répandu d'un jardin japonais -, une petite pierre de fond, oku ishi 奥石, était adjointe aux deux roches principales et d'une petite pierre de devant, mae ishi 前石, était associée à la roche invitée. Le dispositif conservait ainsi l'équilibre initial, mais pour une composition de deux groupes de trois et de deux roches.

15. Les documents, numérotés selon l'ordre de l'alphabet japonaisいろは, permettent de localiser les courettes avec précision dans le plan du palais, grâce au report des caractères japonais sur le «Plan illustré des jardins du palais de Zelkova » 梘御殿庭絵図, un document non daté (réf. 61239), mais qui montre l'état du palais l'agrandissements réalisé pour l'installation de Naonaka. 
La courette présente en outre un assemblage de sept bacs à fleur, ohana oke 御花 槽, disposés en enfilade afin de former une perspective d'alignements de fleurs. Cet élément de décor, rare au Japon ${ }^{16}$, est composé de longs bacs rectangulaires, peutêtre creusés dans de la pierre, ce que les documents ne permettent pas de déterminer avec précision. Dans ce petit jardin, deux alignements de bacs forment un premier plan, le pin parasol constitue le plan central, et les cinq autres bacs sont disposés en arrière-plan.

À la gauche du pin, prend place une vasque de pierre, cylindrique, dont la forme rappelle celle des vasques en forme de maison lunaire, enseishuku 円星宿. Le paysage est encadré par deux haies sèches, shiba-gaki 柴垣 ${ }^{17}$. Sur la droite du dessin, la clôture est dite 《du monastère Kenninji », Kenninji-gaki 建仁寺垣, ce qui désigne une clôture faite de lamelles de bambou disposées verticalement, obtenues en fendant un bambou en quatre. À l'aide d'une corde de couleur brune, tissée à partie de fibres de palmier de Chine (Trachycarpus), dite shuro-nawa 棕櫊縄, les lamelles de bambou sont nouées à une ossature de montants en bambous et en bois. Le dessus de la clôture est protégé avec un assemblage de bambous horizontaux, tama-buchi 玉縁. L'origine du nom vient d'un modèle de clôture qui se trouvait à l'origine au monastère Kenninji de Kyōto. Plusieurs exemples de Kenninji-gaki sont présentés dans l'Iwagumi sono.u yaegaki-den 石組園生八重垣伝 (Enseignement sur les assemblages de pierres, les jardins et les huit épaisseurs de haies) ${ }^{18}$, publié par Akisato Ritō 秋里離 島 (?-vers 1830) en 1827 , ce qui confirme qu'il s'agit là d'un décor commun à cette époque.

En fond de jardin, un réservoir d'eau (tame 溜) est dissimulé derrière une clôture dite en forme de 《fouet à thé », chasen-gaki 茶䇮垣, ou en forme « d'épi » takehogaki 竹穂垣, appelée ainsi en raison de la technique de nouage de rameaux de bambous, dont la partie haute des fins branchages est laissée en forme de plumeau. Une technique que l'on nomme aussi en japonais aori-dome あおり止め, «coupe-vent». Sur le dessin, la clôture porte la mention aoyama-gaki 青山垣, «clôture de la montagne verdoyante », un nom évoquant la haie-sèche d'un ermitage bâti au milieu d'un mont boisé.

Comme l'indique l'inscription okozashiki no ma mae oniwa 御小座敷之間前御 庭, le document marqué du caractère «ろ» dépeint l'aménagement de la cour sud du petit salon du pavillon de Monseigneur. Dans ce jardin, trois larges pierres d'un pas japonais mènent depuis la coursive de l'édifice à un petit bassin circulaire, dont le pourtour est parsemé de pierres plates et de deux roches dressées. Un premier plan, à la droite du dessin, est formé d'un bouquet de trois arbustes : un pin vertical, derrière lequel s'étend la branche d'un vieux prunier, et que précède un pied de nanten 南天

16. On en trouve un exemple similaire dessiné sur les anciens plans du parc Kôrakuen 後楽園, au château d'Okayama.

17. En japonais, la haie sèche, shiba-gaki, est distinguée de la haie vive, ike-gaki 生け垣. Le caractère 垣 désigne à l'origine un mur peu élevé, mais il est utilisé au Japon pour transcrire le mot kaki, qui a le sens de « haie », « clôture ».

18. Voir Uehara Keiji 上原啓二 (éd.), Iwagumi sono.u yaegaki-den 石組園生八重垣伝 (Enseignement sur les assemblages de pierres, les jardins et les huit épaisseurs de haies), coll. Zōen kosho sōsho, vol. 3, Tōkyō, Kajima shoten 1984, 108 p. (noté infra, Yaegaki-den). 
(Nandina domestica). La clôture en treillage, yotsume-gaki 四つ目垣, montre un style commun dans un jardin de l'époque d'Edo (1603-1867), fait de poteaux en bois de section ronde, auxquels sont attachés des bambous noués horizontalement et verticalement, afin de former un treillage régulier. Les bambous verticaux sont coupés de deux hauteurs différentes : disposées à intervalle régulier, les hauteurs donnent une forme dentelée à la partie supérieure de la clôture.

Le document marqué du caractère «は», intitulé «belle vue», kōken 高見, dépeint le chemin d'accès à la maison de thé, osukiya. Il montre la vue depuis l'extérieur vers le pavillon, et non depuis la coursive de l'édifice vers le jardin comme dans le cas des neuf autres dessins. Selon ce document, la cour du pavillon de Monseigneur est couverte de sable blanc, comme l'indique la mention shirasuna (ou hakusa) 白 砂. Une porte rustique ouvre sur un pas japonais. Il s'agit d'une porte en bois de style intermédiaire, gyo no kido 行之木戸, dont la forme simple se distingue de celle d'une porte de style formel surmontée d'un pin en guise de bon augure, matsu no kado 松 の門 ${ }^{19}$. De part et d'autre du pas japonais, des arbustes bas taillés en boule ornent le jardin de thé, alors que d'autres, de dimensions plus imposantes, revêtent les formes de parallélépipèdes et de cylindres caractéristiques de l'art topiaire des jardins seigneuriaux de l'époque. Des pierres cylindriques ou des troncs d'arbres d'enneigement, sekisetsu 積雪, ont été enfoncés dans le sol afin de délimiter un parterre. Sur le côté de l'entrée du pavillon, une vasque à eau, chōzu-bachi 手水鉢, creusée dans une imposante roche, est disposée à l'abri d'une haie-manche, sode-gaki 袖垣, faite de rameaux de bambou. Les haies sèches montrent des roseaux noués en botte aux hauteurs variées.

Le document marqué du caractère «に» décrit le jardin aménagé qui s'étend jusqu'en bordure du lac Matsubara, au nord du pavillon des Tremblements de terre. Deux grands arbres, dont un pin, ont été conservés en fond de jardin, dont ils forment l'arrière-plan. Le motif le plus singulier de cet aménagement est la forme irrégulière de la clôture qui dessine des courbes et des angles, et s'enroule en fond de jardin autour d'une lanterne et du vieux pin. Dans la première courbe, la haie sèche est de type Kenninji, en lamelles de bambou, puis elle devient une « haie striée du Kenninji », shima Kenninji-gaki 嶋建仁寺垣, en raison d'un jeu de lamelle tantôt sombres, tantôt claires, qui donne ainsi l'effet de stries. L'arrondi autour de la lanterne est en treillage, yotsume. En fond de jardin, la clôture est une « haie de bambou en forme d'épi », de type aoyama, sans doute placée en bordure du lac pour son effet coupe-vent. Le motif central de ce jardin est une lanterne de pierre pour regarder la neige, yukimi sekitōrō 雪見石燈籠, posée sur une roche plate ornée d'un rochermaître, lui-même adjoint de deux pierres de côté, et que parsèment quelques bambous noirs.

Le jardin du document marqué du caractère «ほ» est aménagé sur la face ouest du pavillon des Tremblements de terre. En fond de courette, à gauche (côté sud) depuis la vue principale, une clôture en forme de tubes, teppō-gaki 鉄砲垣, ferme le jardin. Selon le Yaegaki-den, la forme de tube peut être réalisée à l'aide de troncs brûlés

19. Iwagumi sono.u yaegaki-den, p. 38. 
(yakimaruta 焼丸太), de gros bambous (ōtake 大竹), de roseaux à flèches (yagaratake 矢柄竹), de tiges de lespedèze (hagi 萩), Lespedeza thunbergii, ou de simples roseaux (yoshi 芦) noués en bottes. Le fond du jardin est fermé par une treille dite « de chemin de montagne », yamamichi-gaki 山道垣, dont la partie haute présente la forme vallonnée d'une cime montagneuse. Les autres côtés du jardin sont clos d'une haie sèche en forme de treillage, yotsume-gaki, où est percée une porte donnant sur une pierre de seuil. Deux groupes de pierres, l'un à gauche, l'autre à droite de la vue principale, structurent le paysage. Différents arbres y sont associés, mais le nom des plantations n'est précisé ni sur ce dessin, ni sur les neuf autres.

Le jardin de la courette au sud du salon privé de Monseigneur correspond au document marqué du caractère " $へ$ ». Il s'agit d'une cour rectangulaire, dont la forme régulière est atténuée par les motifs de clôtures, dont l'un, au premier plan à gauche, est en forme de «tubes », teppō-gaki. Le dessin montre distinctement de fines tiges nouées en bottes, semblables à des tubes disposés les uns à côté des autres. Sur la droite, l'accès de l'édifice depuis la coursive se fait par deux pierres de seuil aux formes naturelles, que protège une « clôture-dos de chameau », rakuda-gaki ラク夕゙垣 - parce que sa découpe arrondie évoque la forme des bosses d'un chameau - et derrière lequel pousse un grand pin. Deux clôtures encadrent de part et d'autre le motif central : un bassin rectangulaire, sensui 泉水, ceint de deux bosquets d'arbustes. Un vieil arbre pousse à l'arrière du bassin.

Le dessin marqué du caractère «と» montre le jardin aménagé devant le pavillon du Salon du Fond, oku goza no ma. Le motif principal est un cours d'eau tortueux, qui coule de la droite vers la gauche, le long de la coursive du pavillon. Les clôtures de type Kenninji présentent des lamelles de bambou d'une hauteur variable. En fond de jardin, la forme douce en arc de cercle de la haie tranche avec les coins anguleux des édifices et des cours. Deux portes y sont percées, afin d'accéder au jardin du pavillon à étage, ochin, l'une, sur la gauche, à double battant avec une couverture en chaume, kaya-mon 萱門, l'autre à simple battant, faite d'une treille de bambous en losanges. De part et d'autre de la porte, la haie sèche est en roseaux, comme l'indique l'inscription ashi-gaki 臀垣. Le chemin d'accès à la porte est un pavement, ishi-jiki 石敷, de type nobe-dan 延段, en pierres aux formes naturelles.

Le cours d'eau est alimenté par une source qui jaillit abondamment depuis un baquet en pierre, placé à l'abri d'un bosquet de cinq arbustes. Le bosquet dissimule un puit en bois, sans doute un regard d'où l'on pouvait doser le débit de la source. De part et d'autre du ruisseau, un sable blanc recouvre les berges. Du côté du pavillon, des pierres plates permettaient de s'approcher au plus près de l'eau sur la rive proche $\mathrm{du}$ pavillon, alors que des pierres semblables à des roches naturelles tapissent les abords de l'autre rive. La partie centrale du cours d'eau est traversée par un ponceau en rondins de bois, maruta-bashi 丸太橋, disposé en diagonale. Trois pins de tailles différentes ornent l'autre rive et masquent à moitié une lanterne pour regarder la neige. Deux groupes de pins verticaux et de dimensions plus imposantes encadrent le paysage de la rivière. Celui de droite, abrite une lanterne « dont la forme rappelle le goût de Sōeki », Soeki-gata 宗易形, c'est-à-dire une forme appréciée par le maître de thé Sen no Rikyū 千利休 (1522-1591). Le dessin montre des treillages de bambou disposés sur les bords du cours d'eau, afin d'y faire pousser des plantes d'eau. 
Le dessin marqué du caractère «ち» porte l'inscription oku goza no ma nishi no hō oniwa 奥御座の間西の方お庭, « jardin donnant sur le côté ouest du pavillon du Salon du Fond ». Ce jardin, l'un des plus vastes, est encadré de trois corps d'édifices : au nord, le pavillon à étage (ochin), à l'est, le pavillon du Salon du Fond, et, au sud, un petit corps d'édifice à l'usage de la famille du seigneur. Le motif central du jardin est un large cours d'eau, sensui 泉水, d'où émergent des îlots de roches dressées. Les berges découpent un bord de mer vallonné et herbeux, parsemé ici et là de pierres, et que bordent tantôt des troncs de bois enfoncés dans l'eau, tantôt des assemblages de petits bois. Le cours d'eau est ici un prolongement du ruisseaux tortueux du précédent jardin (voir supra, dessin «と»).

Devant le pavillon, une pierre pour se déchausser, kutsunugi ishi 沓脱石, à la forme d'un long parallélépipède, permet l'accès à la coursive. Le premier plan de la composition paysagère est un vieux pin tortueux au tronc incliné. L'arbre prend racine au milieu de cinq roches, selon le dispositif évoqué plus haut d'une triade associée à une paire, et il a été taillé afin d'obtenir un étirement de ses branches au-dessus de l'eau. On dit alors nagashi matsu 流松, pin étiré, mais le terme n'est pas noté sur le document. L'arbre abrite un petit autel, dont la structure est visible sur le plan du palais. Trois boules d'arbustes, peut-être des azalées, se mêlent à des roches assemblées autour du pied du tronc. À l'arrière du pin, une avancée sur l'étang, telle une presqu'île, abrite une lanterne en pierre. Sur la rive opposée du cours d'eau, une pierre plate marque l'avancée d'un pont jeté vers un petit îlot couvert de mousse. La partie nord de l'étang (à droite du dessin) présente des groupes de pierres dressées et assemblées en triade ou par groupes de cinq (soit une triade associée à une paire). Les clôtures et les portes sont en forme de treillage, yotsume-gaki, des treillages en losanges pour les portes et en carrés pour les clôtures, avec des hauteurs de montants irrégulières.

Les aménagements paysagers des deux courettes au sud du pavillon du Salon du Fond sont dessinés sur les documents marqués des caractères «り» et «ぬ». Sur le premier («り»), un pas japonais part de la pierre pour se déchausser et se divise en deux branches, formant un arc de cercle. La branche de gauche conduit à un petit bassin en pierre rempli d'eau, de forme carrée, qu'abrite un arbre principal, un arbre secondaire, une boule d'azalée et une pierre dressée. De l'autre côté, le pas conduit à une lanterne en pierre, qu'abrite un grand arbre et trois petits arbres secondaires. La pierre pour se déchausser permet d'accéder sur le côté à une vasque de pierre, dont le dispositif rappelle en tout point celui d'un natsume-gata chōzu-bachi 霜形手 水鉢, une 《vasque en forme de jujube », tel que le décrit le Tsukiyama niwatsukuri-den 築山庭造傳 (Enseignement sur l'aménagement des jardins en montagnes artificielles ${ }^{20}$, publié en 1828 par Akisato Ritō. La gravure de cet ouvrage montre le bord d'une coursive, engawa, et un plancher égouttoir, nure-en 濡縁, destiné à recevoir les écoulements de l'eau puisée dans la vasque. La vasque, à la forme d'un jujube (le fruit du jujubier commun), repose sur une pierre de socle, dai-ishi 台石. Devant elle, au premier plan, une pierre lisse aux reflets chatoyants est dite pierre du miroir dormant,

20. Voir Uehara Keiji 上原啓二 (éd.), Tsukiyama niwatsukuri-den (kōhen) kaisetsu 築山庭造伝 (後編) 解 説 (Enseignement sur l'aménagement des jardins en montagnes artificielles [seconde partie], édition commentée), coll. Zōen kosho sōsho, vol. 2, Tōkyō, Kajima shoten 1965, p. 52. 
kagami ishi 蟄石. Le côté droit de la composition est fermé par une roche cristalline, $s h o ̄ j o-$-ishi 清浄石. Sur la gauche, une grande pierre plate permet de se tenir debout sur le côté de la vasque : c'est la pierre pour puiser l'eau, mizukumi ishi 水汲石. Une autre roche, plus épaisse que les autres et disposée à l'arrière, permet de remplir la vasque, mizuage ishi 水揚石. Au niveau du sol, une sorte de plateau à rebord retient l'écoulement de l'eau (tataki shitsukui nari 湿埽なり). Quelques galets y sont disposés. L'aménagement de la vasque en fore de jujube est isolé du reste du jardin par une clôture-manche, sode-gaki 袖垣, à l'arrière de laquelle se trouvent une lanterne et un pin. Ces éléments, décrits avec minutie dans le Tsukiyama niwatsukuri-den, se retrouvent dans le jardin de la courette, dans lequel la clôture-manche a été remplacée par une haie-sèche de type teppō-gaki, faite de bottes de fines tiges de lespédèzes ou de roseaux. Un arbuste d'arrière-plan laisse entrevoir à son pied une touffe de prèle d'hivers, tokusa 砥草 ou 木賊 (Equisetum hyemale). La lanterne est à l'arrière-plan, plus éloignée de la vasque que celle décrite dans le manuel d'Akisato.

Le second document (dessin «ぬ») porte l'inscription Oku goza no ma nanpō higashi no koniwa 奥御座之間南方東小庭, «jardinet de la courette est, aménagée au sud du Salon du Fond ». Très différent des autres aménagements paysagers, le jardinet est organisé autour d'une pièce d'eau, plus ou moins circulaire, dont le contour est dessiné par un rebord de maçonnerie, ce qui est peu commun au Japon. Trois imposantes pierres plates permettent de s'en approcher et cinq autres sont disposées sur son pourtour. Plusieurs roches dressées saillent au milieu du bassin, où s'accrochent quelques mousses et de rares plantes grimpantes. La disposition des roches se fait par groupes de 3, 5 et 7 éléments. Des arbustes taillés en boules en parsèment le bord et l'arrière-plan.

L'étude des quatre séries de documents relatifs à l'aménagement des cours intérieures du palais privé a montré que la cour de sable blanc n'était pas un motif répandu dans une résidence privée seigneuriale de la fin de l'époque d'Edo. Le long de chaque édifice noble, on trouvait un jardin orné de roches, de cours d'eau, de bassins, de plantes, d'arbustes à fleur, et équipé d'un mobilier composé de vasques et de lanternes en pierre, de haies sèches à motifs de décor faits de bois, de bambous ou de roseaux. L'abondance des bassins et de plans d'eau était rendue possible grâce à un réseau de canalisations d'alimentation, car seule une eau courante permettait de ne pas attirer à proximité des habitations les moustiques et autres insectes nuisibles. Dans cette pratique, la cour d'entrée et la cour devant le salon de réception, ainsi que les cours de service et celles des pavillons du personnel de maison demeuraient couvertes de gravier. Les jardins adoptaient des dispositifs simples et conformes aux usages en vogue à la même époque dans les jardins de Kyōto. Ils témoignaient d'une large diffusion de pratiques et de modèles paysagers plus ou moins stéréotypés, dont on retrouve les descriptions dans les manuels de l'art des jardin publiés par Akisato Ritō à la même époque. 